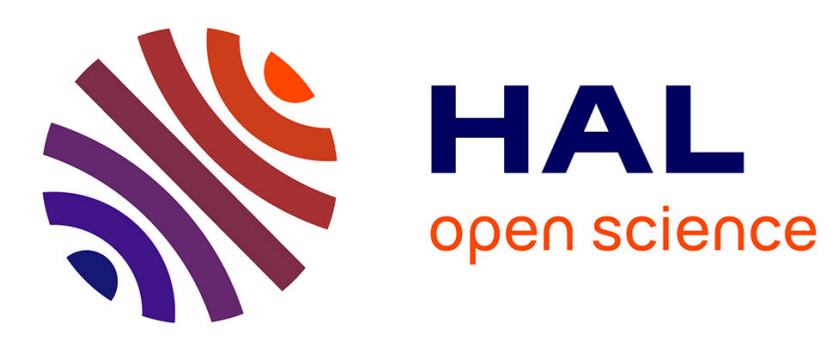

\title{
Comparison of two approaches for differentiating full-field data in solid mechanics
}

\author{
Stéphane Avril, Pierre Feissel, Fabrice Pierron, Pierre Villon
}

\section{To cite this version:}

Stéphane Avril, Pierre Feissel, Fabrice Pierron, Pierre Villon. Comparison of two approaches for differentiating full-field data in solid mechanics. Measurement Science and Technology, 2010, 21 (1), pp.015703. 10.1088/0957-0233/21/1/015703 . emse-00509118

\section{HAL Id: emse-00509118 \\ https://hal-emse.ccsd.cnrs.fr/emse-00509118}

Submitted on 18 Jan 2011

HAL is a multi-disciplinary open access archive for the deposit and dissemination of scientific research documents, whether they are published or not. The documents may come from teaching and research institutions in France or abroad, or from public or private research centers.
L'archive ouverte pluridisciplinaire HAL, est destinée au dépôt et à la diffusion de documents scientifiques de niveau recherche, publiés ou non, émanant des établissements d'enseignement et de recherche français ou étrangers, des laboratoires publics ou privés. 


\title{
Comparison of two approaches for differentiating full-field data in solid mechanics
}

\author{
Stéphane Avril ${ }^{1}$, Pierre Feissel ${ }^{2}$, Fabrice Pierron ${ }^{3}$, Pierre \\ Villon $^{2}$ \\ ${ }^{1}$ Department of Health Science and Engineering \\ Ecole Nationale Supérieure des Mines \\ CNRS UMR 5146 - INSERM IFR 143 \\ 158 Cours Fauriel, 42023 SAINT-ÉTIENNE cedex 2 \\ ${ }^{2}$ Laboratoire Roberval de Mécanique, UTC, \\ BP 20529 rue Personne de Roberval 60205 COMPIEGNE \\ ${ }^{3}$ Laboratoire de Mécanique et Procédés de Fabrication \\ Arts et Métiers Paristech \\ BP 508 rue Saint Dominique 51006 CHÂLONS EN CHAMPAGNE \\ E-mail: pierre.feissel@utc.fr
}

\begin{abstract}
.
In this study, the issue of reconstructing the gradients of noisy full-field data is addressed within the framework of solid mechanics. Two approaches are considered, a global one based on Finite Element Approximation (FEA) and a local one based on Diffuse Approximation (DA). For both approaches, it is proposed to monitor locally the filtering effect in order to adapt the uncertainty to the local signal to noise ratio. Both approaches are applied to a case study which is commonly considered as difficult in solid mechanics (open-hole tensile test on a composite laminate). Both DA and FEA are successful for detecting local subsurface damage from the measured noisy displacement fields. Indications are also provided about the compared performances of DA and FEA. It is shown that DA is more robust, but the downside is that it is also more CPU time consuming.
\end{abstract}

Keywords: full-field measurements, numerical differentiation, measurement uncertainty. 


\section{Introduction}

In experimental solid mechanics, it is required to measure deformations for characterizing the response of solids to a given load. Classically, a strain gauge bonded onto the solid surface provides an average value of the strain across the gauge area. This is particularly adapted to mechanical tests where the stress field is homogeneous in the zone of the gauge area (abusively called homogeneous tests). Indeed, for these tests, the deduced stress/strain curves directly represent the constitutive behaviour of the investigated material. For more complex mechanical tests, the poorness of strain gauge data has lead experimentalists to consider more sophisticated measurement techniques.

In the recent past, the development of full-field optical techniques (FFOTs) to measure kinematic quantities over a whole surface of interest has shed a new light on the mechanical tests for material characterization $[1,2]$. In the framework of in-plane problems, the majority of FFOTs provide full-field displacement measurements, such as digital image correlation [3], speckle interferometry [4], moiré interferometry [5] and grid methods [6]. Only few of them are actually able to provide full-field strains directly, which is the case for shearography techniques $[7,8]$. Details about all these methods can be found in [9].

Each method has advantages and drawbacks and is eventually more or less adapted to a given situation. Among them, a technique that seems to be adequate for mechanical testing is the grid method [10], because it can be applied at a large range of solid materials, be adjusted to study different fields of view by changing the pitch of the grid and it is less sensitive to object vibrations than interferometric techniques.

However, most of the users of full-field measurement methods require strain fields instead of displacement fields to study the phenomena occurring at different scales in the tested materials. Therefore, except for the shearography techniques, it is necessary at some stage in the process to differentiate the data. When the gradients of the measured displacement fields are relatively low, for example when the materials still behave elastically or just beyond the elastic limit, small errors on measurement values at specified points may induce large errors on the computed derivatives [11]. So the key issue is to develop stable algorithms, in which it is possible to quantify explicitly the effects induced by noise on differentiation.

A large number of algorithms can be found in the literature. A survey of these methods is briefly presented in [12]. The most common way of differentiating numerical data is the finite difference method [12]. Simple and effective with precise data, it is implemented in most of the softwares providing full-field displacement data. However, when the level of noise is significant, filtering is required.

The differentiation issue is actually wider than just a noise filtering problem. Full-field measurement methods can provide matrices of data representing more than 100,000 points across a given surface. Therefore, full-field measurement methods provide very densely distributed measurements of the deformation field across a given surface. However, as dense as these measurements can be, they remain discrete. The general 
issue is then: how to reconstruct continuously the deformation fields and its gradients from these discrete and noisy data?

One widely used way of doing this consists in interpolating or approximating the data using smooth basis functions. The differentiation of the data is then simply the differentiation of the basis functions, which is more effective. For a given basis of functions, the regularization parameter is tied to the number of functions used in the basis. The difficulty in controlling this regularization parameter lies within a good compromise between the faithfulness of the reconstruction (obtained with a large number of basis functions) and the efficiency of the low pass filtering (obtained with a small number of basis functions).

However, a good choice of the basis functions is essential [13]. The basis functions can be defined either globally [14], or locally [15]. Previous studies [16] showed that basis of polynomial functions defined globally tend to induce parasitic oscillations in the reconstruction when the degree of the polynomials used in the basis reaches a given threshold. Indeed, because of the global nature of this type of basis, accuracy cannot be reached for both the displacement and its gradient. Accordingly, it seems that basis functions which have limited interactions between each other would be more suitable.

Two approaches fulfilling this requirement were compared in a previous study [17]. The first approach is based on global least-squares minimization using FiniteElement Approximation shape functions as the basis functions (FEA) [18]. The second approach is based on local weighted least-squares minimization using a polynomial diffuse approximation (DA) [19].

After the comparative study published in [17], an issue still remained to be solved for applying these approaches to the open-hole tensile test carried out on quasi-isotropic composite laminates $[16,20]$. This issue is how to control locally the uncertainty of the reconstruction. The regularization parameter is the mesh size for the FEA approach, the span of the weighting function in the DA approach. The open-hole tensile test on composite laminates is widely documented in the literature [7,21-25]. Experimentally, it is a difficult case because the strain field contains at the same time zones with large gradients near the hole and zones with small gradients away from the hole [17]. Therefore, on the one hand, if a large regularization parameter is used to increase the filtering effect, the reconstruction of the concentrated gradients near the hole will be affected. On the other hand, if the regularization parameter is too small, spurious gradients will occur because of the presence of the high spatial frequency noise.

In this paper, in order to address the issue of controlling locally the uncertainty of the reconstruction, an improved regularization approach is implemented in the FEA and the DA methods. Improved means that the regularization parameter is adapted locally. The larger the norm of the local gradients, the smaller the regularization parameter. Accordingly, heterogeneous distributions of the regularization parameters will be used for the FEA and the DA methods. In other words, the filtering effect will be tuned differently at each reconstruction point, as a function of the local signal to noise ratio.

Before presenting the details of these novel approaches, the principle and the 
efficiency of the FEA and the DA methods, which was presented by the same authors in [17], will be detailed and their behaviour with respect to both filtering and approximation will be discussed. Then a criterion will be defined for tuning locally the regularization parameter. The improved versions of the FEA and the DA methods, considering non uniform regularization parameters, will then be presented and studied as applied to the open-hole tensile test. The performances of the methods are compared to help future users choosing what method is the most appropriate to a given field reconstruction problem. Eventually, the two methods are used to detect the local nonlinearities in a composite open-hole specimen.

\section{The reconstruction methods}

\subsection{Framework and illustrative example}

2.1.1. Framework The proposed approaches are aimed at deriving strain fields from a set of full-field displacement measurements. These measurements are derived from the deformation of a pattern attached to the investigated solid, for instance using digital image correlation [3] or fringe analysis [10]. These methods yield the displacement $\underline{u}$ through its coordinates $\left(u_{x}, u_{y}\right)$ on two perpendicular directions (e.g. Figure 1) on a regular grid of data points, defining the measurement zone denoted $\Omega$. From a standard CCD camera of $1200 \times 1000$ pixels, DIC or fringe analysis yields a grid of data points typically from 100 to 200 points per side (therefore from $10^{4}$ to $4 \times 10^{4}$ data points). The input data for deriving strain fields across $\Omega$ are therefore the displacements given at the $N$ data points and are denoted as:

$$
\underline{\tilde{u}}\left(\underline{x}_{i}\right)=\underline{u}_{e x}\left(\underline{x}_{i}\right)+\delta \underline{u}_{(}\left(\underline{x}_{i}\right) \quad, i \in[1, N]
$$

where the $\underline{x}_{i}$ 's denote the geometric positions where measurements $\underline{\tilde{u}}$ are obtained and its coordinates are denoted $\left(x_{i}, y_{i}\right) . \underline{u}_{e x}$ is denoted as the exact vectorial field of displacements and $\delta \underline{u}$ is also a vectorial field representing the measurement error. The sources of uncertainty in optical measurements are numerous because of the complex measurement chains. However, several studies showed that a significant part of the measurement error is composed of an additive uncorrelated white noise $[6,26]$. The present paper is focused on the effects of this additive uncorrelated white noise, denoted as $\delta \underline{u}$, the measurement random error.

In the case of real experimental data, $\underline{u}_{e x}$ is not known, whereas it is known when dealing with synthetic data. In the case of real measurement, $\underline{u}_{e x}$ is actually the sum of the true field and the systematic error of the full-field algorithm. The latter depends on the chosen algorithm and some of them furnish some error estimators [27], but its study is not in the scope of the current paper which focuses on the transformation from displacement to strain, once the displacement is obtained. The displacements considered here are two-dimensional vectors as full-field measurements are almost always performed across flat surfaces and the component of the displacement normal to the surface is usually negligible. The case when the surface is not flat or the case when the component 
of the displacement normal to the surface is not negligible are beyond the scope of this paper. Indeed, the derivation of strains in these cases requires particular kinematic assumptions [28], whereas this paper is fully dedicated to data processing and noise filtering.

The objective is to derive the gradients of $\underline{u}_{e x}$ from $\underline{\tilde{u}}$, while limiting the influence of $\delta \underline{u}$, and to obtain strains as close as possible to the exact ones.

In the whole paper, only infinitesimal strains are considered. As a reminder, in solid mechanics, the infinitesimal strain tensor $\underline{\underline{\epsilon}}$ is defined as the symmetric gradient of the displacement vector $\underline{u}$, which means:

$$
\underline{\underline{\epsilon}}=\frac{1}{2}\left(\nabla \underline{u}+\nabla^{t} \underline{u}\right)
$$

In the notation used here, the tensorial strain components in the $\left(\vec{e}_{X}, \vec{e}_{Y}\right)$ basis are represented in a vectorial format, using the classical convention [29]:

$$
\underline{\epsilon}=\left[\begin{array}{l}
\epsilon_{X X} \\
\epsilon_{Y Y} \\
\epsilon_{X Y}
\end{array}\right]
$$

In the following, the set of values of $\underline{\tilde{u}}\left(\underline{x}_{i}\right), \underline{u}_{e x}\left(\underline{x}_{i}\right)$ and $\delta \underline{u}\left(\underline{x}_{i}\right), \forall i \in[1, N]$, are stacked together and denoted as columns which are respectively $\{\tilde{u}\},\left\{u_{e x}\right\}$ and $\{\delta u\}$. In this study, as the noise is assumed additive and uncorrelated, $\{\delta u\}$ is a random vector and its covariance matrix is:

$$
\operatorname{cov}(\{\delta u\})=\gamma^{2}[\mathcal{I}]
$$

where $[\mathcal{I}]$ is the identity matrix.

2.1.2. Illustrative examples In the following sections, two types of data will be used : experimental data and synthetic data.

Concerning the experimental data, they were obtained on a real quasi-static tensile test carried out onto a glass/epoxy composite laminate [16]. The specimen used in the tests is shown in Figure 1, the dashed zone being the zone where displacement are measured. It is a $4 \mathrm{~mm}$ thick glass-epoxy specimen with the following stacking sequence $\left[-45_{4} / 90_{4} / 45_{4} / 0_{4}\right]_{S}$. Displacement fields were measured using the grid method [10]. A periodical pattern (grid) with crossed lines regularly spaced by $0.1 \mathrm{~mm}$ was transferred onto the surface of the specimen. A CCD PCO PixelFly 12 bits camera, with $1360 \times 1024$ pixels, was used for capturing images of this grid across a surface of $21.5 \times 16.7 \mathrm{~mm}$ located around the hole. Grid images were analyzed using a dedicated algorithm based on the theory of spatial phase shifting [10]. The obtained displacement matrices have a size of $226 \times 170$ data points, each component being an independent measurement corresponding to one period of the pattern (so the assumption of uncorrelated white noise is satisfied). The data grid spacing is about $9.510^{-2} \mathrm{~mm}$. An example of measured displacements is shown in Figure 2. 


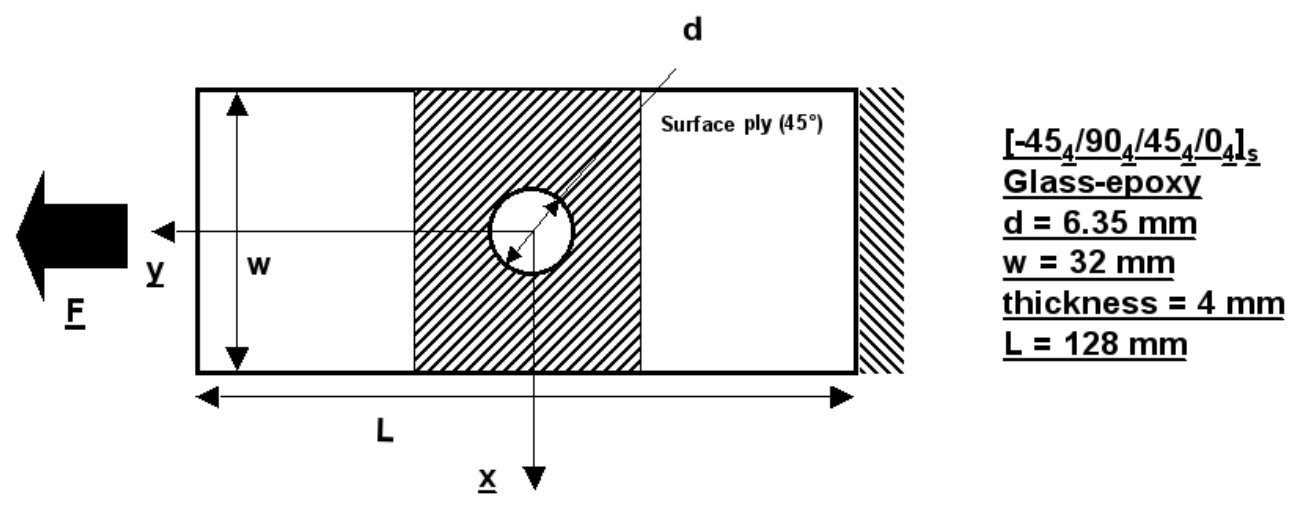

Figure 1. Schematic of the open-hole tensile test.

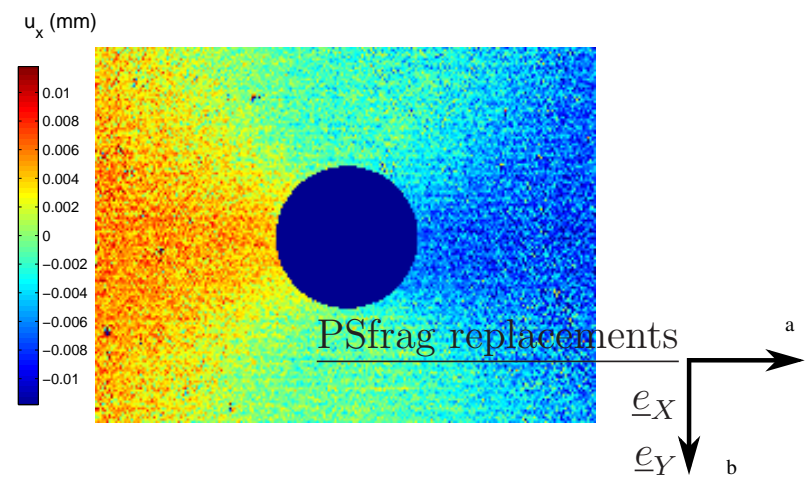

(a) Displacements along $x$

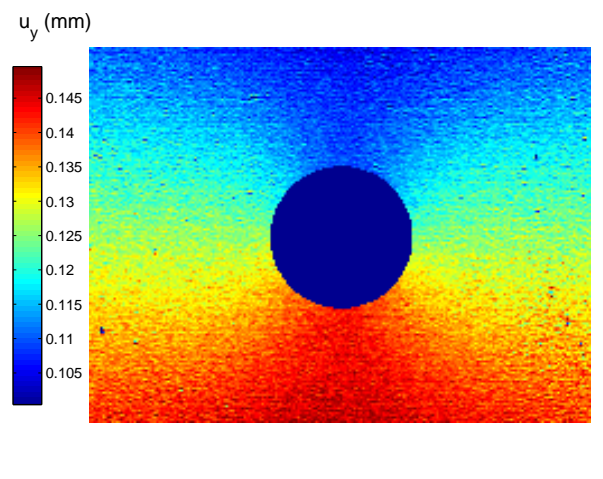

(b) Displacements along $y$

Figure 2. Examples of measured displacement fields.

In this paper, most of the examples are based on synthetic data. The interest of synthetic data is that the exact field $\underline{u}_{e x}$ and the measurement random error $\delta \underline{u}$ are known and one can evaluate properly the reconstruction errors, which is the absolute error of the strain reconstruction process. The synthetic data were obtained from the numerical simulation of a plate tested in an open-hole tensile configuration similar to the one described for the experimental data above, in terms of size and geometry. The simulation is computed using standard plane stress linear 3-nodes elements with an isotropic material, with 150000 nodes, so that the Finite Element fields, which will be considered as the exact ones, are realistic. In order to create the measurements, the Finite Element displacement field is evaluated on a regular grid of $224 \times 168$ data points, by interpolating the nodal displacement with the Finite Element shape functions. The data grid spacing is similar to the experimental one, and therefore equal to about $9.510^{-2} \mathrm{~mm}$. Some noise can be added to simulate the measurement random error, $\delta \underline{u}$. 


\subsection{First reconstruction approach: global least squares/finite element approximation}

A first group of methods consists in using global least-squares minimization over the whole measurement zone $\Omega$. The choice of the basis functions on which the measurements will be projected will affect the regularity and the precision of the reconstruction. A Finite Element Approximation (FEA) [18], where the basis functions have only very low interactions between each other, is chosen in this study because it limits reconstruction oscillations as the precision increases, contrarily to polynomial basis functions [16]. The regularization parameter is hence the meshsize of the FEA, denoted $h$.

The reconstructed displacement field is written like this:

$$
\underline{u}_{a p}(\underline{x})=[\phi(\underline{x})]\{U\}
$$

where $[\phi(\underline{x})]$ is the elementary matrix of the shape functions evaluated at $\underline{x}$ and $\{U\}$ is the column of the nodal displacements. For a given mesh, one has only to determine $\{U\}$ to define the reconstructed field.

In the present example, the elements are triangles with linear shape functions [17]. Let $[\Phi]$ be the matrix made up of all the matrices $\left[\phi\left(\underline{x}_{i}\right)\right]$ stacked together, $i \in[1, N]$. $[\Phi]$ collects the shape functions evaluated at the data points. It can be written:

$$
\left\{u_{a p}\right\}=[\Phi]\{U\}
$$

where $\left\{u_{a p}\right\}$ is hence the column made up of all the values of $\underline{u}_{a p}\left(\underline{x}_{i}\right), i \in[1, N]$.

$\{U\}$ is found as the solution of the following minimization:

$$
\min _{\{U\}} \sum_{i=1}^{N}\left\|\left[\phi\left(\underline{x}_{i}\right)\right]\{U\}-\underline{\tilde{u}}\left(\underline{x}_{i}\right)\right\|^{2} \Leftrightarrow \min _{\{U\}}\left([\Phi]^{t}\{U\}-\{\tilde{u}\}\right)^{t}\left([\Phi]^{t}\{U\}-\{\tilde{u}\}\right)(7)
$$

The minimization problem (7) leads to a linear system to be solved, yielding $\{U\}$ :

$$
\{U\}=[S]^{-1}[\Phi]^{t}\{\tilde{u}\}
$$

where:

$$
[S]=[\Phi]^{t}[\Phi]
$$

$[S]$ is a sparse matrix, with nonzero components located close to the diagonal.

The strain field can be directly derived from $\underline{u}_{a p}(\underline{x})$, by differentiating the shape functions. It can be written:

$$
\underline{\epsilon_{0}}(\underline{x})=[b(\underline{x})]\{U\}
$$

where $[b(\underline{x})]$ collects the components of the symmetric gradient of $[\phi(\underline{x})]$ and $\epsilon_{0}(\underline{x})$ is the strain field evaluated at $\underline{x}$. By collecting the strain at the data points, it can be written:

$$
\left\{\epsilon_{0}\right\}=[B]\{U\}
$$


where $\left\{\epsilon_{0}\right\}$ is the column made up of all the values of $\underline{\epsilon}_{0}\left(\underline{x}_{i}\right), i \in[1, N]$, and $[B]$ is the matrix made up of all the matrices $\left[b\left(\underline{x}_{i}\right)\right]$ stacked together, $i \in[1, N]$.

For the type of applications targeted here, the $\underline{\epsilon_{0}}(\underline{x})$ field is not suitable as a reconstructed strain field because it is discontinuous. In order to ensure the continuity of the approximated strain field, a second strain field, denoted $\underline{\epsilon}_{a p}(\underline{x})$, is reconstructed as a linear combination of the $\phi(\underline{x})$ shape functions, such as:

$$
\underline{\epsilon}_{a p}(\underline{x})=[\phi(\underline{x})]\{E\}
$$

where $\{E\}$ is the column of nodal strains. It means that the strain field $\underline{\epsilon_{0}}$ is projected onto the basis of functions $\phi(\underline{x})$. Nodal values $\{E\}$ are the solution of the following global least-squares problem:

$$
\min _{\{E\}} \int_{\Omega}\|[b(\underline{x})]\{U\}-[\phi(\underline{x})]\{E\}\|^{2} d \Omega
$$

In (13), the integrals are estimated using finite element numerical integration, with one Gauss point per element and leads to matrices similar to mass or rigidity matrices. This minimization problem leads to a linear system to be solved, yielding $\{E\}$ :

$$
\{E\}=[S]^{-1}\left[S^{\alpha}\right][S]^{-1}[\Phi]^{t}\{\tilde{u}\}
$$

where: $\left[S^{\alpha}\right]=[B]^{t}[\Phi]$.

\subsection{Second reconstruction approach: diffuse approximation/polynomial approximation}

A second group of methods is based on the use of local regression [15]. The Diffuse Approximation (DA) [19] has been initially developed to solve partial differential equations (PDE), as an alternative to finite elements or finite difference methods. It has been used in several fields, from field transfer [30] to optimization through response surfaces [31]. Here, it is chosen as an approximation operator because it offers a wide range of possibilities, in terms of function basis or in terms of span of the local regression. Furthermore due to its orientation towards the solving of PDE, its theoretical behaviour has been thoroughly studied, which is a clear advantage.

The function basis of the DA is chosen as polynomials with various degrees. As this approach is based on weighted least-squares, a key point is the span of the weighting function, denoted $R$. This parameter can be tuned to obtain the best regularization/precision compromise. As presented below, the (diffuse) derivatives are directly derived from the measurements with this approach.

The key point of the DA is to define a continuous field from a discrete number of data points through the following method. Let us consider a basis of functions, here monomials, that are collected in a line vector $p(\underline{x})$. For example, for a degree 2 basis, one has:

$$
p(\underline{x})=<1 \quad x \quad y \quad \frac{x^{2}}{2} \quad x y \quad \frac{y^{2}}{2}>
$$


The diffuse field is then defined as:

$$
u_{a p}(\underline{x})=v(\underline{x}, \underline{x}) \quad \text { where, } \quad v\left(\underline{x}, \underline{x}^{\prime}\right)=p\left(\underline{x}^{\prime}-\underline{x}\right)\{a(\underline{x})\}
$$

where $\{a(\underline{x})\}$ is a vector of parameters depending on $\underline{x}$ that will be determined by the local least squares associated with $\underline{x}$. The introduction of function $v$ is not mandatory but allows to present the centered Diffuse Approximation whose interpretation is directly correlated to the Taylor series around the reconstruction point [19]. For a degree 1 basis, $v\left(\underline{x}, \underline{x}^{\prime}\right)$ writes:

$$
v\left(\underline{x}, \underline{x}^{\prime}\right)=a_{1}(\underline{x})+a_{2}(\underline{x})\left(x^{\prime}-x\right)+a_{3}(\underline{x})\left(y^{\prime}-y\right)
$$

For any reconstruction point $\underline{x}, a(\underline{x})$ is then defined as the solution of the following local weighted least-squares problem across the neighbourhood $V(\underline{x})$ of $\underline{x}$ :

$$
\min _{a(\underline{x})} \frac{1}{2} \sum_{\underline{x}_{i} \in V(\underline{x})} w\left(\underline{x}, \underline{x}_{i}\right)\left(p\left(\underline{x}-\underline{x}_{i}\right)\{a(\underline{x})\}-\tilde{u}\left(\underline{x}_{i}\right)\right)^{2}
$$

$\underline{x}$ being a constant with respect to the minimization and the function $w\left(\underline{x}, \underline{x}_{i}\right)$ being the weighting function evaluated at each data point. This can be rewritten in a matrix form as follows:

$$
\min _{\{a\}} \frac{1}{2}[[P]\{a\}-\{\widetilde{U}\}]^{T}[W][[P]\{a\}-\{\widetilde{U}\}]
$$

where $[P]$ is the matrix collecting the $p\left(\underline{x}_{-} \underline{x}_{i}\right), \underline{x}_{i} \in V(\underline{x}),\{\widetilde{U}\}$ the column collecting the points data in $V(\underline{x})$ and $[W]$ is the diagonal matrix with $W_{i i}=w\left(\underline{x}, \underline{x}_{i}\right)$.

$w\left(\underline{x}, \underline{x}_{i}\right)$ can be any positive function defined over a bounded domain. The bounded domain requirement is aimed at keeping the local character of the reconstruction. Among several possibilities, the weighting function is defined as follows, because of the square grid of data points:

$$
w\left(\underline{x}, \underline{x}_{i}\right)=w_{r e f}\left(\frac{x-x_{i}}{R_{x}}\right) w_{r e f}\left(\frac{y-y_{i}}{R_{y}}\right)
$$

where $w_{r e f}$ is a dimensionless window function whose derivative zeroes at 0 and 1 (this aspect ensures continuity up to the first derivative of the reconstructed fields), [17]; here $R_{x}$ and $R_{y}$ are chosen independent of $\underline{x}_{i}$ but may depend on $\underline{x}$. Despite it is not mandatory, $R_{x}$ and $R_{y}$ are taken equal and denoted $R$, corresponding to the span of the weighting function.

Then, $\{a(\underline{x})\}$ as the solution of (18) is given by:

$$
\{a\}=\left[P^{T} W P\right]^{-1} P^{T} W\{\widetilde{U}\}
$$

If the basis is composed of monomials up to at least degree 1, the approximate field and its first derivatives are reconstructed as the first three components of vector $\{a\}$ :

$$
u_{a p}(\underline{x})=a_{1}(\underline{x}), \quad \frac{\delta u_{a p}}{\delta x}(\underline{x})=a_{2}(\underline{x}) \quad \text { and } \quad \frac{\delta u_{a p}}{\delta y}(\underline{x})=a_{3}(\underline{x})
$$

where $\frac{\delta u}{\delta x}$ denotes the diffuse derivative which is an approximation of the exact derivative. This reconstruction can be applied to each component of the measured displacement 
field $\underline{\tilde{u}}\left(\underline{x}_{i}\right)$. The strain fields can be deduced subsequently from the first order diffuse derivatives. Let us finally mention that this approach implies the resolution of problem (18) at each evaluation point. This means that one has to choose the evaluation points. This choice depends on the application of the method, here, they are chosen as coincident with the data points where data are provided. Accordingly, the reconstruction is evaluated through the discrete grid of the points $\underline{x}_{i}, i \in[1, N]$.

In summary, the parameters to be tuned in the DA method are the degree of the polynomial basis and the span of the weighting function, $R$. As discussed in [17], a basis with polynomials of degree 2 is a good compromise between the filtering effect and the faithfulness of the reconstruction, as the filtering is almost as efficient as for a basis of polynomials of degree 1 and the faithfulness of the reconstruction is improved. Therefore degree 2 is used in the following. Only $R$, the span of the weighting function, will be considered as tuning parameter for controlling the uncertainty in the following.

\section{Reconstruction errors - effect of the filtering parameters ( $h$ or $R$ )}

\subsection{Splitting of the error: approximation error and random error}

The reconstructed field $\underline{u}_{a p}(\underline{x})$, at any $\underline{x}$, can be split up into three parts as follows:

$$
\underline{u}_{a p}(\underline{x})=\underline{u}_{e x}(\underline{x})+\delta \underline{u}_{k}(\underline{x})+\delta \underline{u}_{b}(\underline{x})
$$

where $\delta \underline{u}_{k}(\underline{x})$ corresponds to the error due to the approximation of the exact field and can be seen as the systematic error of the strain reconstruction operator. $\delta \underline{u}_{b}(\underline{x})$ is the random error implied by the noise on the measurements. In the following, $\delta \underline{u}_{k}(\underline{x})$ is called the reconstruction error, $\delta \underline{u}_{b}(\underline{x})$ is called the random error and $\delta \underline{u}_{k}(\underline{x})+\delta \underline{u}_{b}(\underline{x})$ is called the absolute error. The same splitting is achieved on the reconstructed strain field:

$$
\underline{\epsilon}_{a p}(\underline{x})=\underline{\epsilon}_{e x}(\underline{x})+\delta \underline{\epsilon}_{k}(\underline{x})+\delta \underline{\epsilon}_{b}(\underline{x})
$$

Since the reconstruction operators are linear, the approximation error $\delta \underline{u}_{k}(\underline{x})$ is related to the reconstruction of the exact field alone and the random error $\delta \underline{u}_{b}(\underline{x})$ is exactly the reconstruction of the noise alone.

Obviously, such splitting cannot be achieved with real data because the exact field is not known in experiments. Nevertheless, one objective of this study is to understand and control the reconstruction error, therefore the numerical example is useful. Since the evaluation of the approximation error is not straightforward, we will focus on the random error in the applications and discuss the limitations of the approach. As suggested in Section 2.1.2, the actual noise can reasonably be modeled realistically by a white noise (4), so it is possible to bound the term $\delta \underline{u}_{b}(\underline{x})$ and then determine the local uncertainty. This will be presented afterwards. 


\subsection{Effect of $h$ or $R$ on the reconstruction quality}

First we illustrate the effect of the mesh size $h$ (for FEA) and of the span of the weighting function $R$ (for the DA) on the reconstruction quality and the balance between the magnitude of $\delta \underline{\epsilon}_{k}(\underline{x})$ and $\delta \underline{\epsilon}_{b}(\underline{x})$ using synthetic data. In this case, the exact field is simulated as described in Section 2.1.2, so it is known. The reconstruction absolute error can be deduced straight forwardly. In order to assess it, the following criterion, based on the true error, has been defined at any $\underline{x}$ :

$$
e_{\epsilon}(\underline{x})=\sqrt{\left(\epsilon_{a p_{X X}}-\epsilon_{e x_{X X}}\right)^{2}+2\left(\epsilon_{a p_{X Y}}-\epsilon_{e x_{X Y}}\right)^{2}+\left(\epsilon_{a p_{Y Y}}-\epsilon_{e x_{Y Y}}\right)^{2}}
$$

This criterion is the norm of the absolute error of the three components of the strain tensor.

Then, a mean error over any area $\Omega^{\prime}$ of $N^{\prime}$ reconstruction points is defined as:

$$
e_{\epsilon}^{\text {mean }}\left(\Omega^{\prime}\right)=\frac{1}{N^{\prime}} \sum_{i=1}^{N^{\prime}} e_{\epsilon}\left(\underline{x}_{i}\right)
$$

Two reconstructions are performed. The first one from measurements without noise, yielding the approximation error alone. The second one is performed on a white noise alone yielding the random error. The simulated white noise is Gaussian, with a standard deviation similar to the one deduced from the experiments, about $5 \%$ of the mean displacement of the studied case.

frag replacements ERROR MAP EF

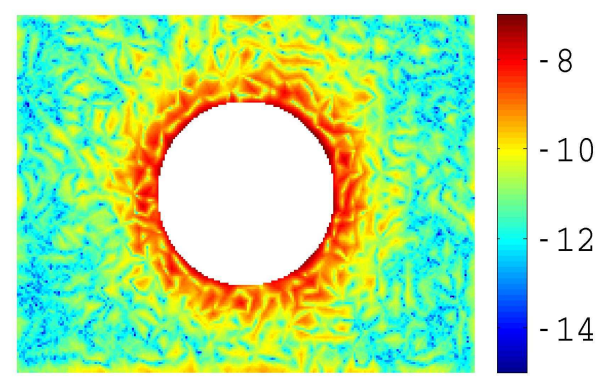

(a) Approximation error - logscale

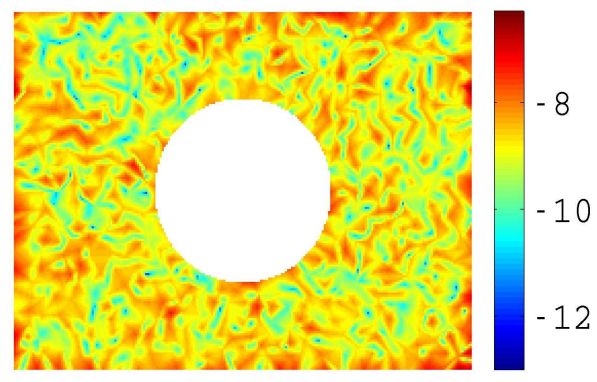

(b) Random error - logscale

Figure 3. Error Map - FEA - $h=6$ data points

Figure 3 and 4 present the error maps both in terms of random error and approximation error, for the two approaches with standard values of $h$ and $R$ ( $h=6$ and $R=16$, in number of data points) ; these values are chosen based on the previous study [17] and yield a good balanced between the two types of error on our example as will be confirmed by Figure 6. One can note that for both cases, the approximation error is located near the hole where large gradients occur. Concerning the FEA, the reconstruction is affected by the mesh, another mesh with the same mesh size would imply a slightly different reconstruction. Since the noise magnitude is constant all over the domain, the random error is globally uniform in magnitude. Nevertheless, typical 
frag replacements RROR MAP DA

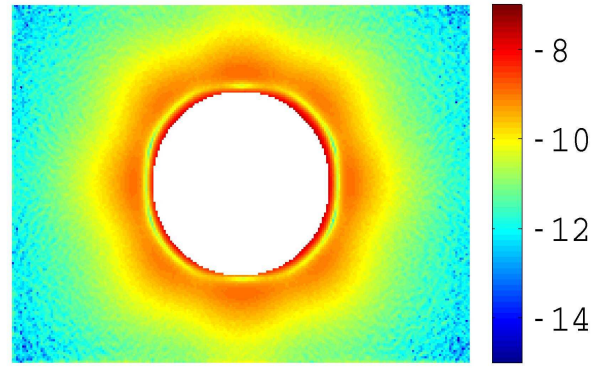

(a) Approximation error - logscale

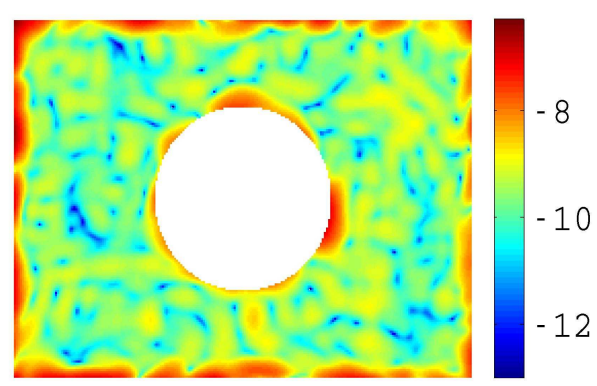

(b) Random error - logscale

Figure 4. Error Map - DA - $R=16$ data points

patterns appear with a characteristic length directly related to the value of $h$ or $R$ and one can note that the error increases on the edges of the domain; this will be explained in Section 3.4.

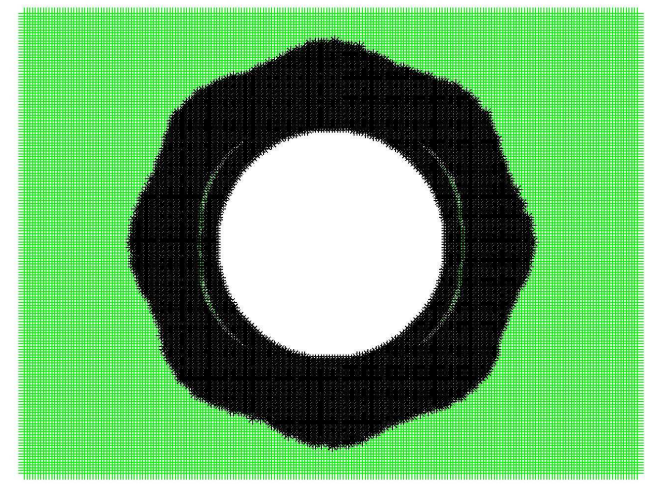

Figure 5. Selected zone around the hole for the mean error $e_{\epsilon}^{\text {mean }}$

Results showing the values of the criterion $e_{\epsilon}^{\text {mean }}$ on an area around the hole with and without noise have been plotted in Figure 6. The aera is made up of 9821 data points represented on Figure 5. It can be observed that for small values of $h$ or $R$, the term $\delta \underline{\epsilon}_{k}$ (approximation error) is almost negligible compared to $\delta \underline{\epsilon}_{b}$ (random error). When $h$ or $R$ increases, both types of error tend to be balanced, and, beyond, $\delta \underline{\epsilon}_{k}$ overtakes $\delta \underline{\epsilon}_{b}$.

In this section, a first example was used to illustrate the separation of errors and how they are affected by $h$ and $R$. The choice of values for these parameters will be the result of a compromise between the two types of error. In order to estimate the approximation error, one would need to construct an error estimator, which does not seem straightforward. Concerning the random error, one can characterize it from a 
frag replacements noise alone with noise without noise Radius $R$ $e_{\epsilon}^{\text {mean }}$

Measurements :

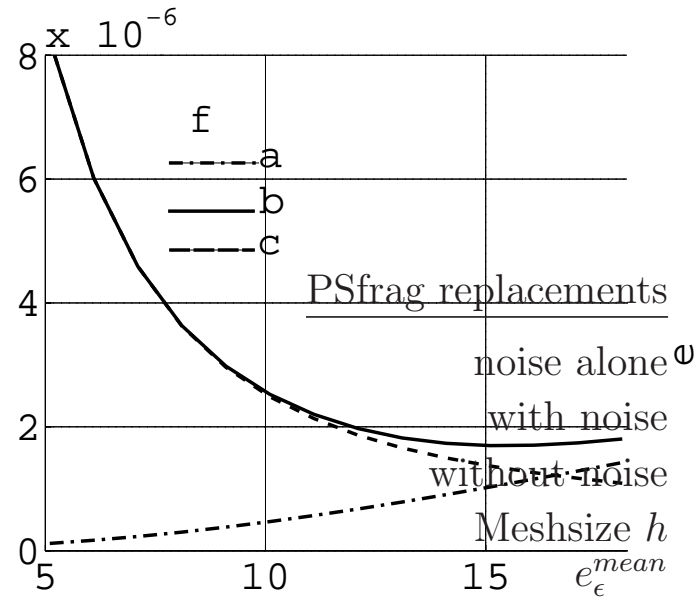

d Measurements :

(a) Diffuse Approximation

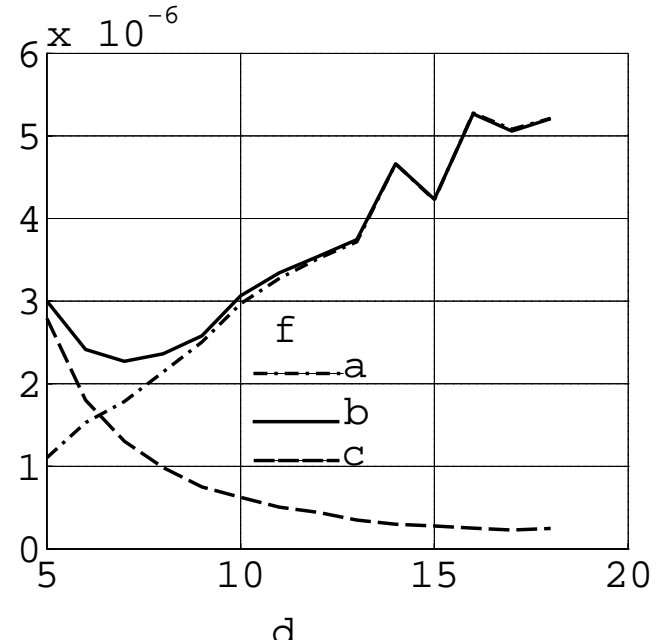

(b) Finite Element Approximation

Figure 6. Error $e_{\epsilon}^{\text {mean }}$ around the hole as a function of $R$ or $h$

statistical point of view. This is described for the FEA and the DA in the following sections.

\subsection{Random error estimation for the FEA method}

The random error is obtained by studying the reconstruction of the noise alone. It can be studied from a probabilistic point of view using the relationship between the standard deviation on the input noise and the standard deviation on the output strain field. The study is first performed on the FEA.

Assuming the measurement noise is a white noise with a covariance defined by (4), $\{U\}$ defined in equation (8) for the FEA approach is a random vector. From (8), its covariance matrix is:

$$
\operatorname{cov}(\{U\})=\gamma^{2}[S]^{-1}
$$

Eventually, $\{E\}$ is also a random vector. Its covariance matrix is:

$$
\operatorname{cov}(\{E\})=\gamma^{2}[S]^{-1}\left[S^{\alpha}\right]^{t}[S]^{-1}\left[S^{\alpha}\right][S]^{-1}
$$

In equations (27) and (28), the off-diagonal terms are at least one order of magnitude lower than the diagonal ones. Therefore, they may be neglected at the first order. Moreover, for nodes away from the boundaries, all the diagonal terms are similar at the first order. Let $\sigma_{U}$ be the average square root of the diagonal terms in $\operatorname{cov}(\{U\})$ and let $\sigma_{E}$ be the average square root of the diagonal terms in $\operatorname{cov}(\{E\})$. From equations (27) and (28), it appears that:

$$
\left\{\begin{array}{l}
\sigma_{U}=\gamma \alpha_{U} \\
\sigma_{E}=\gamma \alpha_{E}
\end{array}\right.
$$


where the factors $\alpha_{U}$ and $\alpha_{E}$ are called the coefficients of sensitivity to noise. The coefficients of sensitivity to noise are functions of the mesh size $h$. Using the definition of matrix $[S]$ in equation (9), it has been deduced that:

$$
\alpha_{U} \cong \frac{2.6}{h}
$$

This equation has been derived by considering that a given node belongs to six elements at the same time. This is only true when the node is away from the edges of the measurement area.

As for $\alpha_{E}$, one obtains the following equation:

$$
\alpha_{E}=\frac{0.12}{p_{\text {unit }} h^{2}}
$$

where $p_{\text {unit }}$ is the spatial resolution of the measurements, i.e. the distance between two adjacent independent data points.

\subsection{Random error estimation for the DA method}

The same study on the random error is performed on the DA reconstruction. By denoting $M_{\epsilon}$ the linear strain reconstruction operator, the reconstructed strain field is given by the following relation:

$$
\underline{\epsilon}(\underline{x})=M_{\epsilon} \widetilde{U}_{x}
$$

where $\widetilde{U}_{x}$ collects the displacements of the data points contributing to the reconstruction at $\underline{x}$.

Assuming that each data set is obtained from a Gaussian white noise with standard deviation $\gamma$ as in (4), the covariance of $\epsilon(\underline{x})$ can be written as:

$$
\operatorname{cov}(\epsilon(\underline{x}))=\left\langle\epsilon(\underline{x}) \epsilon(\underline{x})^{T}\right\rangle=\left\langle M_{\epsilon} \widetilde{U} \widetilde{U}^{T} M_{\epsilon}^{T}\right\rangle=\gamma^{2} M_{\epsilon} M_{\epsilon}^{T}
$$

Therefore, knowing the noise on the measurement, which can be estimated in practice, and the reconstructing operator, one can deduce the standard deviation on the components of the strain.

Knowing the $M_{\epsilon}$ operator, one can therefore estimate the value of the ratio of the output to the input standard deviation (sensitivity to noise). Furthermore, this can be achieved for reconstruction points located anywhere in the neighbourhood. This includes the case of points close to an edge. The effect of $R$ and of the position of the reconstruction point in the neighborhood, denoted as its offset, onto the variance of $\epsilon_{X X}$ are illustrated in Figure 7. It can be observed that the offset of the reconstruction point has an effect on the variance. The variance is minimum when the reconstruction point is located at the center of the neighbourhood, and it increases when its position has an offset with regard to the center. This explains why the random error increases along the edges of the reconstruction domain (Section 3.2). Concerning the effect of $R$, Figure 7 shows that the variance decreases with respect to $R$, with a power law since the log-log plot is linear. 


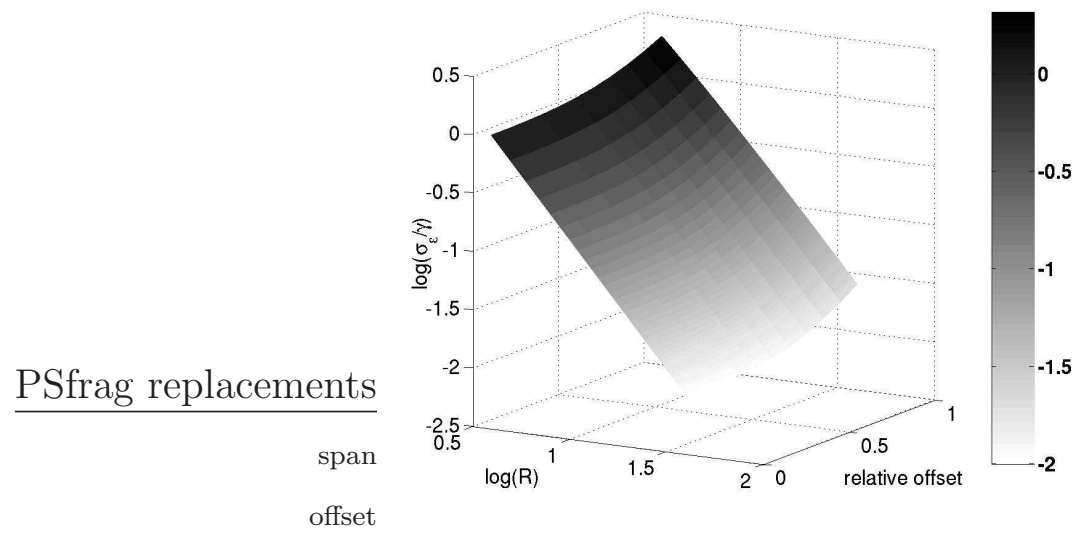

Figure 7. Standard deviation on $\epsilon_{X X}$ as a function of the span $R$ and the offset, logscale

\section{Choice of the reconstruction parameters and related criteria}

\subsection{Choice of a criterion}

In Section 3.2, it was shown that both the random error and the approximation error depend on $\underline{x}$. In the areas where the approximation error remains small, the filtering can be improved by increasing $h$ or $R$. Therefore, an interesting option is to tune these parameters with respect to the space variables. To do so, a criterion has to be established for the selection of $h$ or $R$. According to Figure 6, the absolute error is close to its minimum when both the errors are balanced. A criterion would therefore be to choose $h$ or $R$ such that the random error and the approximation error are equal. However, this implies an error estimator for $\delta \underline{\epsilon}_{k}(\underline{x})$ which is not straightforward and has not been developed yet. Furthermore, concerning the Diffuse Approximation, it can actually be shown that the error is related to the derivatives of higher order than the polynomials of the basis. A major drawback of an estimator of the approximation error is hence that it is based on higher order derivatives, which evaluation needs more care than the first order derivatives that are given by the reconstruction [15].

Nevertheless, an alternative approach is chosen here to derive a criterion. It was observed that in a large number of heterogeneous tests, the areas with large strains are limited in space, therefore they also correspond to zones where the gradient of the strain field is large. In these cases, the zones with high strain values will be the zones where one has to reduce the value of $h$ or $R$ in order to control the approximation error. This leads to define a criterion based on a constant signal to noise ratio (SNR) that is prescribed onto the reconstructed field across the whole measurement area, the objective being to reach a constant relative error all across the reconstruction area. As expected, this criterion will yield larger $h$ or $R$ where the strains are larger and therefore is an improvement with respect to the standard approach where $h$ or $R$ are constant all over the measurement zone. 
First, a signal to noise ratio (SNR) on the reconstructed strain field is defined as:

$$
\frac{\epsilon}{\sigma_{\epsilon}}=\eta
$$

where:

- $\epsilon=\sqrt{\epsilon_{X X}^{2}+\epsilon_{Y Y}^{2}+2 \epsilon_{X Y}^{2}}$. $\epsilon$ is to be estimated. It can be obtained with a first reconstruction, using a strong filtering;

- $\sigma_{\epsilon}$ is defined as: $\sigma_{\epsilon}=\sqrt{\sigma_{X X}^{2}+\sigma_{Y Y}^{2}+2 \sigma_{X Y}^{2}}$, where the $\sigma_{\circ}$ are the standard deviations on the corresponding $\epsilon_{\circ}$ ( (we therefore neglect the coupling effects). $\sigma_{\epsilon}$ is directly related to $h$ or $R$ through the relations (29) and (33) established in Section 3.3 and 3.4 .

Then, the criterion is such that a constant SNR $\eta$ is imposed all over the area. Imposing $\eta$ yields therefore a value of $R$ or $h$ at each position of the measurement area.

For example, by fixing the SNR $\eta$ to 8 , the criterion yields respectively an optimal mesh (figure $8(\mathrm{a})$ ) or a map of optimal influence radii (figure $8(\mathrm{~b})$ ), optimal being defined with regard to the criterion of equation (34). The choice of $\eta=8$ is motivated by the previous study on the absolute error in Section 3.2 and corresponds to the global optimal signal to noise ratio (see Figure 6). On Figure 8(b) the radius map is not symmetric since it is based on noisy data. Note that these meshes or maps of optimal radii are constructed without taking into account the edge effects. To take them into account, the mesh size or the radii should be increased near the edges of the measurement area. Since the key point here is just to illustrate the fact that the $h$ or $R$ parameters can be tuned accross the measurement area, a simple criterion has been used.

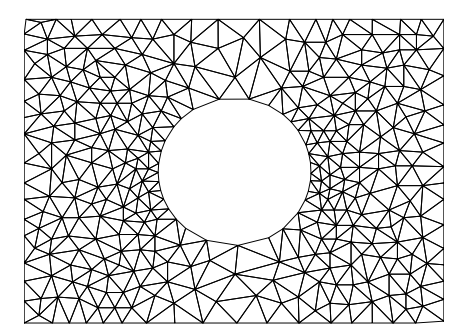

(a) Optimal meshing

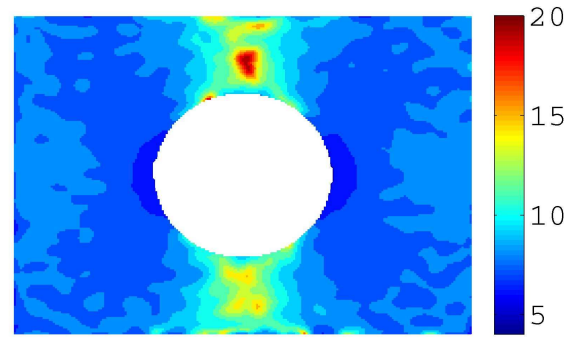

(b) Map of optimal $R$

Figure 8. Optimal $h$ and $R$ for $\eta=8$

\subsection{Practical use of the criterion for an improved reconstruction}

Now, the reconstruction can be achieved by each method with respectively the optimal mesh or optimal radii distribution. The fields reconstructed by both improved approaches have been plotted in Figures $9(\mathrm{~b})$ and $9(\mathrm{c})$. The reconstruction is very promising in comparison to all published material regarding full-field measurements 
on the open-hole tensile test. It seems that for the DA approach, there are parasitic oscillations which are more visible than for the FEA approach. This can be explained by the fact that for the same given standard deviation to achieve, the local zone of influence is much larger for the FEA. The spatial correlation of the reconstructed strain is therefore higher, leading to strains reconstructed from the noise with the same magnitude as with the DA but with smoother spatial variations. From a computation point of view, the FEA method is quicker. But the downside is that it requires a mesh. In each mesh, the mesh size is prescribed but the position of the nodes is random. This means that for each mesh, a different reconstruction will be obtained. The drawback was well illustrated in [17] where the reconstructed data were used for identification purpose, yielding a mesh dependency on the identification results.

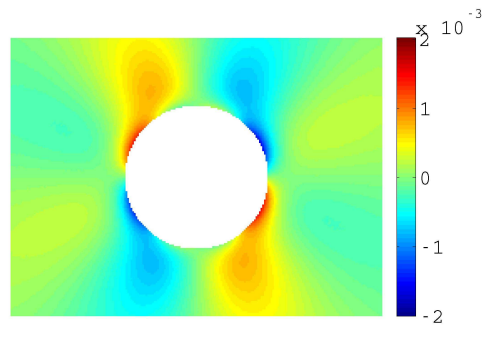

(a) $\epsilon_{X Y}$, exact

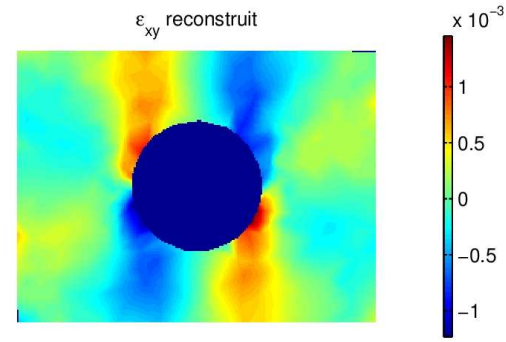

(b) $\epsilon_{X Y}$, FEA, optimized mesh

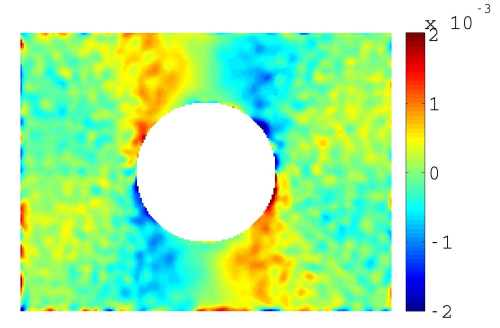

(c) $\epsilon_{X Y}$, DA, optimized radii $R(\underline{x})$

Figure 9. Reconstructed shear strain fields with optimized $h$ or $R$

\section{Application to the detection of subsurface damage in a composite laminate}

In this section, the two reconstruction approaches are applied to data obtained from an open-hole tensile test carried out onto a glass/epoxy laminated plate having a quasi-isotropic stacking sequence: $\left[-45_{4}, 90_{4}, 45_{4}, 0_{4}\right]_{s}$. In such tests, fracture of the $90^{\circ}$ underlying plies occurs quite early. An objective of full-field displacement fields is to detect the onset and spatial location of this fracture. Thirty snapshots of the displacement fields were taken during the test and the grid method [10] used to derive displacements in a region around the hole of the specimen, for tensile loads ranging 
from 0 to $13.3 k N$. It has been proposed in [32] to characterize the fracture process by detecting local non linearities of the surface response. A first estimation of these nonlinearities is obtained by computing the discrepancy between the experimental strain field and the strain field resulting from the extrapolation of the linear response. One has therefore to determine the latter.

The linear response is estimated from the first ten strain maps, $k \in\{1, . .10\}, F_{k}$ being the corresponding load, as the slope of the response at each pixel $\underline{x}$ :

$$
\min _{\epsilon_{\text {lin }}(\underline{x})} \sum_{k}^{N_{\text {lin }}}\left(F_{k} \epsilon_{l i n}(\underline{x})-\epsilon\left(\underline{x}, F_{k}\right)\right)^{2} \quad \text { with, } \quad N_{\text {lin }}=10
$$

where $\epsilon\left(\underline{x}, F_{k}\right)$ is the strain at data point $\underline{x}$ deduced from the response corresponding to the load $F_{k}$. Then, it is possible to define the non-linear part of the strain field for a given load $F$, corresponding to a further strain map:

$$
\Delta \epsilon(\underline{x}, F)=F \epsilon_{l i n}(\underline{x})-\epsilon(\underline{x}, F)
$$

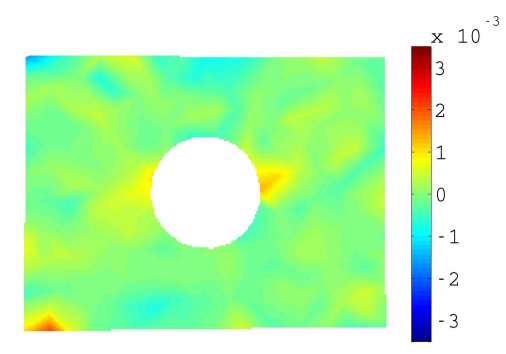

(a) Load $9.7 k N$ - FEA

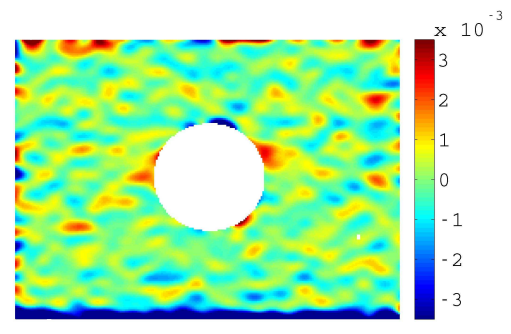

(c) Load $9.7 k N$ - DA

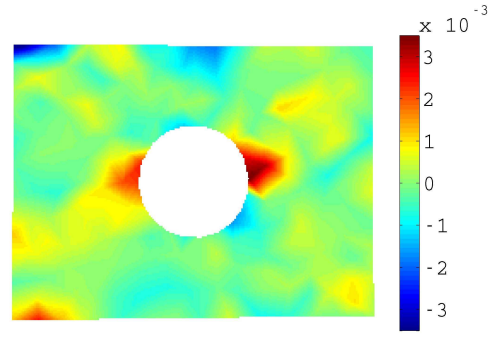

(b) Load $13.3 k N$ - FEA

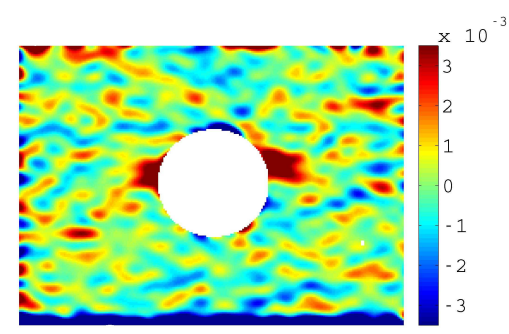

(d) Load $13.3 k N$ - DA

Figure 10. Non-linear part of the displacement from the reconstructed fields

The $\Delta \epsilon$ fields reconstructed by the two approaches are shown for two different loads in Figure 10. The detection of the non-linearities around the hole gives better results than the previous method used in [16], with a sharper zone providing a more precise localization. Both methods yield similar results, even if the FEA approach remains sensitive to the underlying mesh. The DA is more affected by the random error but seems to offer a better contrast. Nevertheless, the perturbations on the measurements are more severe than in the numerical case and one would have to input more a priori 
information on the fields to get better results. This suggests considering regularization approaches, which are considered as one of the major prospects of this study.

\section{Conclusion}

In this paper, two approaches to obtain the strain field from a measured displacement field while controlling the error due to this operation were presented. The first method is based on a global least squares with Finite Element basis functions (FEA) and the second one is based on the Diffuse Approximation (DA). Both methods yield similar results in term of random error and approximation. The FEA is numerically more efficient but its results suffer from a mesh dependency. The DA appears to be richer, with a stronger theoretical background, and offers more evolution possibilities, such as the enrichment of the approximation basis by any physical field from any a priori information.

The key point of this paper is that the filtering parameters ( $h$ or $R$ ) can be tuned across the measurement area. For this purpose, the behaviour of the two approaches was characterized, in terms of approximation and random error. The latter has been studied from a statistical point of view: given the level of noise on the measurement, one can deduce the standard deviation of the reconstructed strain fields. A first reasonable choice for a criterion to define maps of values for parameters $h$ and $R$ was therefore proposed, based on a constant signal to noise ratio on the reconstructed field all across the measurement area. This criterion applied to an example confirms the feasibility of the local tuning of the filtering. It offers wide perspectives of improvement, in particular by defining more accurate criteria, for example based on cross-validation approaches [15].

\section{References}

[1] M. Grédiac. The use of full-field measurement methods in composite material characterization: interest and limitations. Composites Part A: Applied Science and Manufacturing, 35(7-8):751$761,2004$.

[2] A.S. Kobayashi. Handbook on Experimental Mechanics. Wiley, 1993.

[3] H. Schreier and M. Sutton. Systematic errors in digital image correlation due to undermatched subset shape functions. Experimental Mechanics, 42:303-310, 2002.

[4] G.L. Cloud. Optical methods of engineering analysis. Cambridge University Press, Cambridge, 1995.

[5] D. Post, G. Han, and P. Ifju. High sensitivity moiré. Springer Verlag, New York, 1994.

[6] Y. Surrel. Design of algorithms for phase measurements by the use of phase stepping. Applied Optics, 35(1):51-60, 1996.

[7] J.R. Lee, J. Molimard, A. Vautrin, and Y. Surrel. Application of grating shearography and speckle shearography to mechanical analysis of composite materials. Composites Part A, 35:965-976, 2004.

[8] Y.Y. Hung and H.P. Ho. Shearography: an optical measurement technique and applications. Materials Science and Engineering, 49:61-87, 2005.

[9] P.K. Rastogi. Photomechanics. Springer Verlag, Berlin Heidelberg, 2000.

[10] Y. Surrel. Moiré and grid methods: a signal processing approach. In Jacek Pryputniewicz, Ryszard 
J. et Stupnicki, editor, Interferometry '94: Photomechanics, volume 2342, pages 213-220. The International Society for Optical Engineering, SPIE, Nov 1994.

[11] M.G.D. Geers, R. De Borst, and W.A.M. Brekelmans. Computing strain fields from discrete displacement fields in 2D solids. International Journal of Solids and Structures, 33(29):42934207, 1996.

[12] T. Wei and M. Li. High order numerical derivatives for one-dimensional scattered noisy data. Applied Mathematics and Computation, 175:1744-1759, 2006.

[13] F.J. Hickernell and Y.C. Hon. Radial basis function approximations as smoothing splines. Applied Mathematics and Computation, 102(1):1-24, 1999.

[14] I. Lira, R. Cordero, M. François, and C. Vial-Edwards. The uncertainty of experimental derivatives: application to strain measurement. Measurement Science and technology, 15:2381$2388,2004$.

[15] W.S. Cleveland and C. Loader. Smoothing by local regression: principles and methods. Springer, 1995.

[16] F. Pierron, B. Green, and M.R. Wisnom. Full-field assessment of the damage process of laminated composite open-hole tensile specimens. Part I: Methodology. Composites Part A: Applied Science and Manufacturing, 38(11):2307-2320, 2007.

[17] S. Avril, P. Feissel, F. Pierron, and P. Villon. Estimation of strain field from full-field displacement noisy data. Revue Européenne de Mécanique Numérique, 17(5-7):857-868, 2008.

[18] Z. Feng and R.E. Rowlands. Smoothing finite-element and experimental hybrid technique for stress analyzing composites. Computers and Structures, 6:631-639, 1991.

[19] B. Nayroles, G. Touzot, and P. Villon. La méthode des éléments diffus. Comptes rendus de l'Académie des Sciences, série 2, Mécanique, Physique, Chimie, Sciences de l'Univers, Sciences de la Terre, 313(2):133-138, 1991.

[20] A. Balaco de Morais. Open hole tensile strength of quasi-isotropic laminates. Composites Science and Technology, 60:1997-2004, 2000.

[21] S.G. Lekhnitskii, S.W. Tsai, and T. Cheron. Anisotropic plates. New York: Gordon and Breach, 1968.

[22] E.V. Iarve and N.J. Pagano. Singular full-field stresses in composite laminates with open holes. International Journal of Solids and Structures, 38:1-28, 2001.

[23] F. Lagattu, M.C. Lafarie-Frenot, T.Q. Lam, and J. Brillaud. Experimental characterisation of overstress accommodation in notched CFRP composite laminates. Composite Structures, 67(3):347-357, 2005.

[24] J. Molimard, R. Le Riche, A. Vautrin, and J.R. Lee. Identification of the four orthotropic plate stiffnesses using a single open-hole tensile test. Experimental Mechanics, 45(5):404-411, 2005.

[25] S.D. Pandita, K. Nishiyabu, and I. Verpoest. Strain concentrations in woven fabric composites with holes. Composite Structures, 59:361-368, 2003.

[26] M. Bornert, F. Brémand, P. Doumalin, J.C, Dupré, M. Fazzini, M. Grédiac, F. Hild, S. Mistou, J. Molimard, J.-J. Orteu, L. Robert, Y. Surrel, P. Vacher, and B. Wattrisse. Assessment of digital image correlation measurement errors: Methodology and results. Experimental Mechanics, 49(3):353-370, 2009.

[27] G. Besnard, F. Hild, and S. Roux. Finite-element displacement fields analysis from digital images: Application to portevin-le châtelier bands. Experimental Techniques, 46:789-803, 20062006.

[28] E. Soppa, P. Doumalin, P. Binkele, T. Wiesendanger, M. Bornert, and S. Schmauder. Experimental and numerical characterisation of in-plane deformation in two-phase materials. Computational Materials Science, 21(3):261-275, 2001.

[29] S.W. Tsaï and H.T. Hahn. Introduction to composite materials. Editions Technomic Publishing Co., 1980.

[30] D. Brancherie, P. Villon, and A. Ibrahimbegovic. On a consistent field transfer in non linear inelastic analysis and ultimate load computation. Computational Mechanics, page online, 2007.

[31] P. Breitkopf, H. Naceur, A. Rassineux, and P. Villon. Moving least squares response surface 
approximation: formulation and metal forming applications. Computers and Structures, 83(1718):1411-1428, 2005.

[32] F. Pierron, B. Green, M.R. Wisnom, and S.R. Hallett. Full-field assessment of the damage process of laminated composite open-hole tensile specimens. Part II: Experimental results. Composites Part A: Applied Science and Manufacturing, 38(11):2321-2332, 2007. 